\title{
Infrared microscopic analysis of tissues: a comparison of methodologies.
}

\section{Jackson}

Institute for Biodiagnostics, National Research Council Canada, 435 Ellice Ave., Winnipeg, Canada, R3B 1Y6

Given that the infrared spectrum of most materials is one of the most characteristic properties of that material, it seems reasonable to assume that infrared spectra of human tissues will be equally characteristic. This has in fact been shown to be the case, with many reports appearing in the literature over the last 10 years that describe the spectral characteristics of both normal and abnormal tissues (see Figure 1).

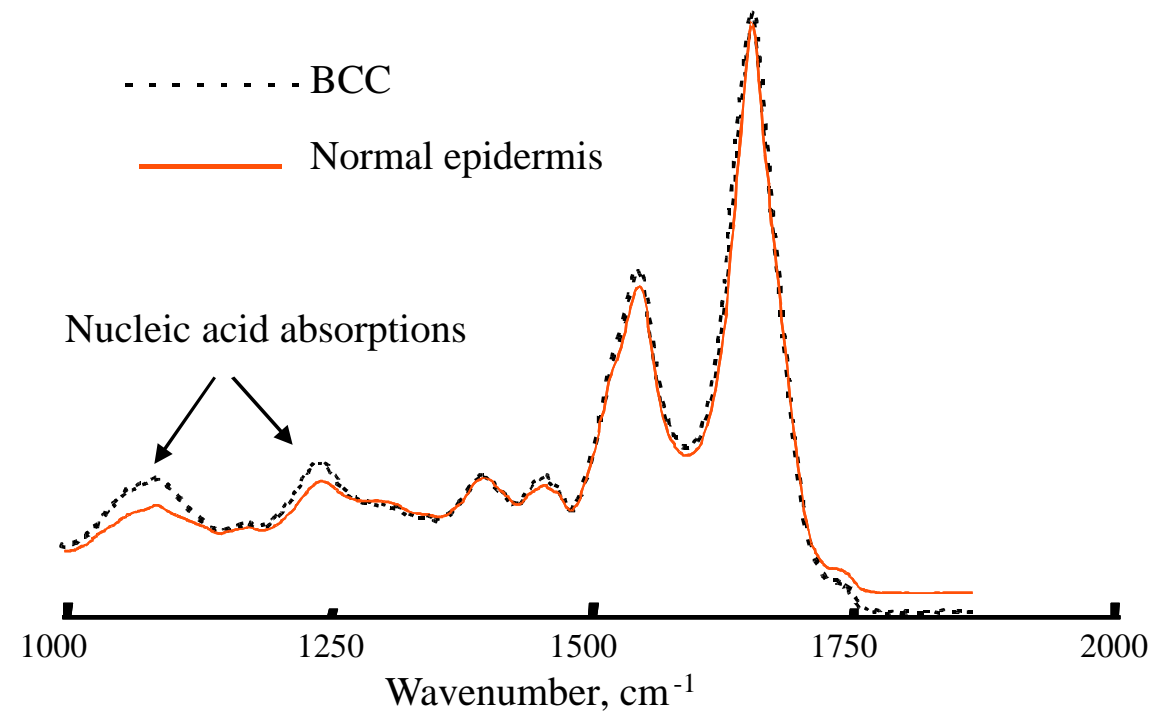

Figure 1. IR spectra of basal cell carcinoma and normal skin.

General agreement is now appearing that IR spectroscopic analysis of tissues is most reliably performed using an infrared microscope. This is based upon the fact that IR microscopy allows tissues to be probed non-destructively with relatively high spatial resolution and direct correlation may be made between spectra and sample histology. Raman spectroscopy offers similar information, but does of course have inherent advantages over infrared techniques in terms of spatial resolution (with distinct disadvantages when spectral quality and data acquisition are compared). However, there is by no means consensus with regards to many of the practical aspects of vibrational spectroscopic analysis of tissues. A number of important questions remain to be addressed before such a consensus can be reached. For example do we really understand biological vibrational spectra? Do we acquire spectra under conditions that give physiologically relevant results? Is the quest for enhanced spatial resolution worth the financial (and temporal) cost (see Figure 2)? Can these techniques ever be clinically relevant or will they remain limited to use in research laboratories? 
This presentation will attempt to address these issues by focusing on the following questions:

1. What are the main chromophores that give rise to useful absorptions in biological systems?

2. How complete is our understanding of vibrational spectra of tissues?

3. What is the most appropriate manner to prepare samples?

a. Is it realistic to analyse tissues that have been stained?

b. Is reflection or transmission the preferred method?

4. Are there true benefits to be obtained from greater spatial resolution (see Figure 1)?

5. What is the most appropriate instrumental approach (standard mapping microscopy, synchrotron based microscopy, microscopic imaging with a focal plane array system)?

6. What are the factors that impede clinical use of vibrational microspectroscopy?

These questions will be addressed from 1) a qualitative spectroscopic standpoint with data acquired from the same set of samples using a variety of methods and 2) a practical standpoint (acquisition time, data quality etc). In addition to comparing data obtained using a variety of infrared microscopic techniques, the presentation will compare data obtaining using IR and Raman instrumentation.
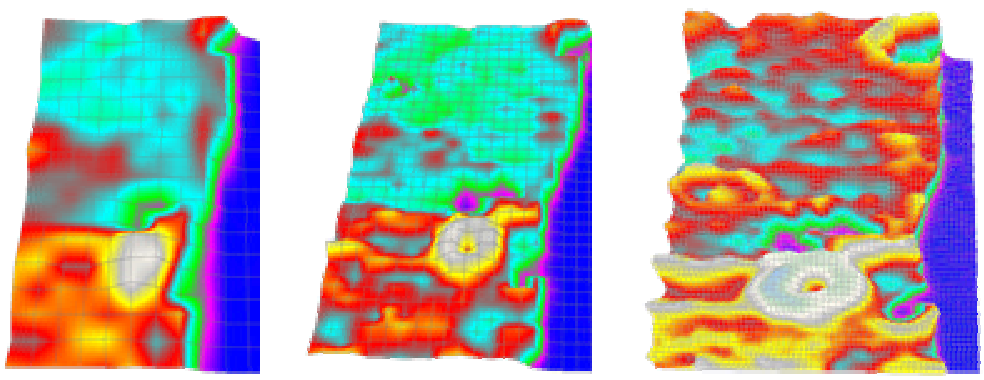

Figure 2. Amide I functional group maps of skin tissue acquired using three different aperture sizes.

\section{References}

M. Jackson, H.H. Mantsch, "Biomedical infrared spectroscopy," in Infrared Spectroscopy of Biomolecules, H.H. Mantsch and D. Chapman (Eds), Wiley-Liss, Inc., Toronto, pp. 311-340 (1996).

M. Jackson, H.H. Mantsch, "Infrared Spectroscopy, Ex Vivo Tissue analysis by" in Encyclopedia of Analytical Chemistry, R.A. Myers (Ed), John Wiley \& Sons Ltd, Chichester pp 131-156 (2000)

G.J. Puppels and J. Greve, "Whole cell studies and tissue characterization by Raman spectroscopy" in Biomedical Applications of Spectroscopy, R.J.H Clark and R.E. Hester (Eds) John Wiley \& Sons Ltd, Chichester, Chapter 1 pp1-47 (1996).

E. A. Carter and H.G.M Edwards, Biological Applications of Raman Spectroscopy" in Infrared and Raman Spectroscopy of Biological Materials H-U Gremlich and B. Yan (Eds) Marcel Dekker, Inc., New York, Chpater 11, pp 421-475 (2000 